\title{
Integrating Embedded Computing Systems Into High School and Early Undergraduate Education
}

\author{
Bridget Benson,
}

Arash Arfaee, Choon Kim, Ryan Kastner,
Rajesh K. Gupta,

and

\begin{abstract}
Early exposure to embedded computing systems is crucial for students to be prepared for the embedded computing demands of today's world. However, exposure to systems knowledge often comes too late in the curriculum to stimulate students' interests and to provide a meaningful difference in how they direct their choice of electives for future education and careers. This paper describes an experience with integrating embedded computing systems education into high school and early undergraduate curricula to give students that needed early exposure. It provides assessment data that illustrates the success and limitations of the efforts described as well as the lessons they hold for a reform of the undergraduate curriculum and its impact on high school education.
\end{abstract}

\section{INTRODUCTION}

A TYPICAL "computer-centric" computer engineering undergraduate program starts with a heavy dose of programming and/or algorithms. This used to be a perfectly natural way to treat undergraduate education since it provides the quickest path for the students to become familiar with computing. However, today an entering computer science undergraduate student already knows what a computer is or what it can do as a consumer device. Mysteries remain as to how computers actually interact with real life. What does it mean exactly when one says there is a computer, or several computers, in a cell phone, an iPod, or a car? How exactly does a computing machine work? How does it interact with other machines and systems? While embedded systems comprise about 99\% of the entire computer market [1], many undergraduate computer engineering programs still teach programming and design skills that are applicable to a general-purpose computer rather than to the more specialized embedded systems [2]; those programs that teach embedded systems courses do so at the upper-division level [3]. The fact that embedded systems are key components used in many industries (including the automotive, consumer electronics, military/aerospace, office automation, telecommunication, and data-communication industries [4]-[6]) and that demands for qualified embedded systems engineers are increasing suggests that students should be exposed to embedded systems concepts earlier on in their education. Early exposure can give young students the interest and initiative to pursue an embedded systems education and take more advanced courses later on in the curriculum that will prepare them for the demands of today's embedded world.

But how should embedded systems concepts be taught to students with very little or no computing experience? This paper describes a multipronged approach to provide young students with opportunities to use and understand embedded systems through hands-on interaction with simple embedded systems design. This includes a four-week summer course that exposes high school students to embedded systems as part of the University of California, San Diego, (UCSD) California State Summer School for Mathematics and Science (COSMOS) program [7], as well as integration of embedded systems programming into the undergraduate sophomore-level course CSE30, "Computer Organization and System Programming." The goals of these courses are to:

1) extend embedded systems knowledge to young students;

2) enhance young students' interests and opportunities in the field.

This paper describes the initial experiences in both the COSMOS program and the CSE30 class. First, Section II describes related programs and addresses some of the challenges associated with teaching computing concepts to novices. Section III provides the course description of COSMOS and CSE30. Section IV provides assessment data that illustrate how well the students met the course goals. The paper concludes in Section $\mathrm{V}$ with a discussion on the lessons learned from the courses and with suggestions for future improvement.

\section{RELATED PROGRAMS}

Teaching embedded systems concepts to young students who lack background computing knowledge can be challenging. Young students often lack the attention span, interest, and discipline to sit through traditional conceptual lectures and read up on concepts they do not understand. However, experience has shown that even young novices can learn about and gain an interest in computing through courses that contain a substantial hands-on component. Courses that have a "hands-on" nature can provide some fun and excitement in a student's learning experience and ensure that s/he will not easily forget what has been learned [8]. Some programs that have been successful in using a hands-on approach to teach embedded computing concepts to young students include the COPIRE, SSEST, LEGO Mindstorms Robots, and IPRE programs.

The Community-based Partnership for Integrated Research and Education (COPIRE) program in Cobb County, GA, involves students in a seven-week All-Hands-On summer 
academy with the goals of extending embedded systems research/knowledge to high school students and enhancing these students' interests and opportunities in the field [9]. Japan's Summer School on Embedded System Technology (SSEST) program is intended for young students already pursuing a computer science education. Its main objective is to solve the problem of the scarcity of embedded system developers in Japan [10] by imparting basic knowledge and techniques of the whole embedded system development process to their young students [11] through a hands-on approach. LEGO Mindstorms Robots [12] and the Institute for Personal Robots in Education (IPRE) [13] also take a hands-on approach, focusing on using robots to make computer science education more fun and effective for all education levels.

Thus, COSMOS and CSE30 focus on providing young students opportunities to use and understand embedded systems through hands-on interaction with simple embedded system design.

\section{COURSE DESCRIPTION}

\section{A. COSMOS}

COSMOS is a four-week residential summer program open to high school students, ranging from those entering 9th grade through those exiting 12 th grade, with demonstrated interest and achievement in math and science. COSMOS students enroll in one of eight academic core content courses called "clusters" for the duration of the four-week program. Each cluster is designed and taught by UCSD faculty, lecturers, researchers, and graduate students.

The summer of 2008 was the first summer an embedded systems "cluster" was offered as part of the COSMOS program. The course was designed around six hands-on embedded systems labs that made use of the Cypress Semiconductor CY3214 and CY3209 Development kits [14], inexpensive development kits that offer all the capability needed to build simple embedded systems. The following six lab assignments were adapted from Cypress tutorials. These labs were supplemented with lectures on embedded systems concepts and the $\mathrm{C}$ programming language to give the students the tools they would need to complete the assignments.

1) Pushbutton and Lights: The objective of this assignment was to introduce students to the CY3209 development board and Cypress's PSOC Designer 5 development environment and PSOC Programmer ver. 3.0. Students learned the design flow of a PSOC board by designing a simple project to use a pushbutton input to control LED outputs. No prior programming experience was necessary as the students could design the entire system through the use of a GUI.

2) I2C Slave and Master Communication: The objective of this assignment was to learn how to design an I2C Master and Slave communication between two PSOC modules. Students had to adjust the potentiometer on the slave and observe the master's multidigit LED display and the slave's LED change in response to the changes in the potentiometer. Again, the entire system was designed through a GUI.

3) Pushbutton Counter Implementation: The objective of this assignment was to learn how to write $\mathrm{C}$ code for an embedded environment and get it to run on the CY3214 de- velopment board. The students had to write code to count the number of pushbutton inputs and alter the LCD display and LEDs based on this count.

4) USB Interface Design: The objective of this assignment was to learn how to design a USB interface between the PSOC CY3214 and a personal computer (PC). The students had to click buttons on a PC client program and see the corresponding LEDs on the CY3214 light up. Students could also manually turn LEDs on or off on the CY3214 and see the corresponding buttons light up on the PC client program.

5) CapSense Input Design: The objective of this assignment was to learn how to incorporate the CapSense button input on the CY3214. The students wrote C code to have the LCD show "Button Pressed" when the CapSense button was pressed and show a sliding bar graph on the LCD as the student moved his/her finger across the CapSense slider.

6) Wireless USB (WUSB) Master and Slave Design: The objective of this assignment was to learn how to design a wireless USB Master and Slave communication protocol between two CY3209 modules. This lab assignment was very similar to Lab \#2 in that the students adjusted the potentiometer on the slave device to observe changes in the master's multidigit LED display and slave's LED.

By the end of the course, each student was expected to have completed each lab assignment and used the knowledge they learned in the lab to develop their own embedded systems group project with one or more of the development boards.

The COSMOS embedded systems course met Monday, Wednesday, and Friday mornings (9 a.m.-12 p.m.) for lecture, and every weekday afternoon (1-4 p.m.) for lab, to ensure ample time for the hands-on laboratory component of the course. During the last week of the course, Monday, Wednesday, and Friday morning sessions were also spent in the lab to give students sufficient time to work on their group projects. Students spent Tuesday and Thursday mornings in a scientific communications class to improve their oral and written communication skills. Most of the students completed the six lab assignments between the second and third week of the course and thus spent one to two weeks on their final group projects. Each group was required to demo their project and create a PowerPoint presentation and poster of their project. They presented their projects to the entire class on the last day of the course and displayed their posters for the COSMOS Open House for their parents, staff, faculty, and community members.

\section{B. CSE30}

CSE30, "Computer Organization and Systems Programming," is a required course for all UCSD computer science undergraduates. It teaches the ability to program in assembly, reinforces $\mathrm{C}$ programming concepts, and provides an introduction to computer organization. The course has two prerequisites: basic Java programming (CSE8 series) and basic data structures (CSE12). CSE30 is a prerequisite for a number of upper-division courses including "Computer Architecture," "Compilers," and "Operating Systems." The course has been a favorite course among undergraduates, taught for the last five years by Mr. Rick Ord, a UCSD lecturer who has received numerous teaching awards. 
In the Winter and Spring quarters of 2009, two variations of CSE30 were offered: CSE30-A, the class in its current form, and CSE30-B, the class augmented with an embedded systems component. CSE30-B included embedded system labs to teach the basic principles of using and programming the Cypress development boards. The CSE30-A courses were taught by Mr. Rick Ord, while the CSE30-B courses were taught by Prof. Ryan Kastner and Prof. Rajesh Gupta in the Winter and Spring quarters, respectively.

The two variations of the courses taught the same basic principles. These include an introduction to computer organization, translations of $\mathrm{C}$ to assembly (and vice versa), and basic memory management. The CSE30-A class focused more on systems programming; it used the SPARC assembly language and involved the integration of $\mathrm{C}$ and assembly code on assignments that run on Sun servers. The CSE30-B class taught MIPS assembly using a simulator and introduced the Cypress embedded systems development boards for programming assignments and final class projects.

\section{ASSESSMENT}

In order to assess the effectiveness of the COSMOS and CSE30 courses on accomplishing the goals presented in Section I, each student was evaluated on their labs and final projects and given a post-course survey. For the 2008 and 2009 COSMOS courses, the survey included five open-ended questions and 12 questions that required a numerical response on a Likert scale (1-Strongly Disagree, 2-Disagree, 3-Neutral, 4-Agree, 5-Strongly Agree) [16]. The survey was distributed to the 2008 class by e-mail 10 months after the course had ended, and was distributed to the 2009 class in person on the last day of their course. Fourteen out of 21 students responded from the 2008 class, and 15 out of 20 students responded from the 2009 class. For the Spring CSE30-A and CSE30-B courses, the survey included four open-ended questions and 11 questions that required a numerical response on the Likert scale. These surveys were distributed as part of the final exam in each course, thus all 38 students in CSE30-A and all 19 students in CSE30-B completed the surveys.

The responses from the questions that required numerical responses on the Likert scale were grouped into three categories: Disagree (Strongly Disagree and Disagree), Neutral (Neutral), and Agree (Strongly Agree and Agree), as a student's extent of agreement or disagreement may vary each time s/he takes the same survey. The percentages of students who agreed, disagreed, or had no opinion (neutral) for each question was determined, and the "average" response was calculated by assigning 0 for Disagree, 0.5 for Neutral, and 1 for Agree. No statistics were computed on the results as the sample size was small. The following sections report an evaluation of the student's labs and final projects and a subset of the numerical results from the surveys.

\section{A. COSMOS}

In the 2008 and 2009 COSMOS courses, all students successfully completed all six lab assignments (described in Section III) and a final group project. The group projects ranged in complexity from small modifications to the lab assignments to entirely new embedded system designs, depending on the skill

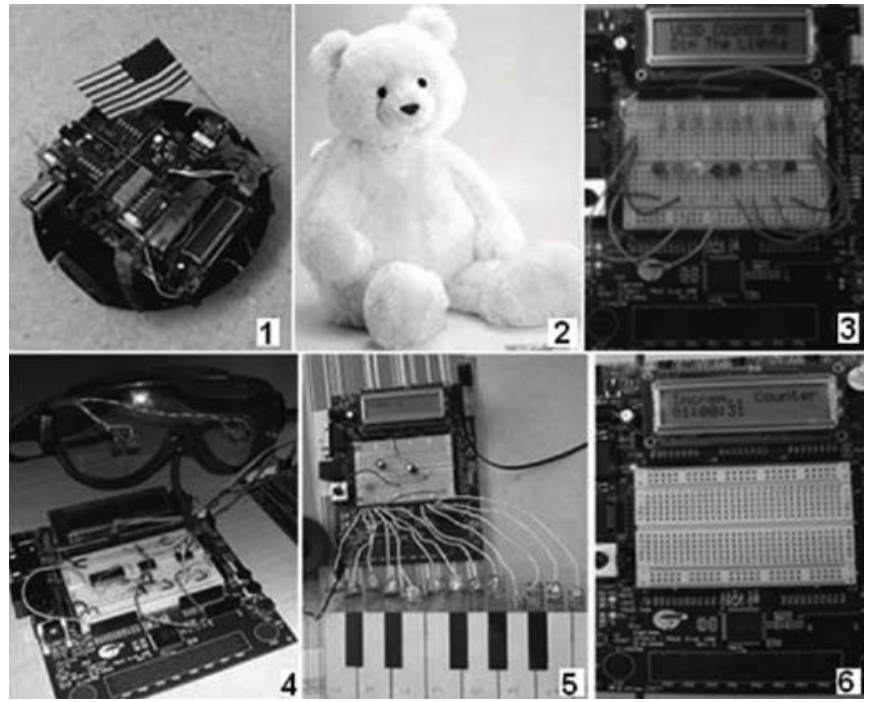

Fig. 1. COSMOS student final group projects.

level of the students in the group. Each student was very proud of the work they had done, and the faculty, staff, and students were all very impressed with the innovation and quality of work the students produced in just a four-week period. The following are a few of the projects designed by the COSMOS students, shown in Fig. 1.

1) Tilt-Controlled Vehicle: The most advanced group of students designed a remote-controlled vehicle, which made use of three CY3209 boards. Two boards made up the "body" of the car and were used to control the servomotors that powered the vehicle. The third board, connected to the "body" via WUSB, was used as the vehicle controller. The students used the accelerometer on the controller board to control the direction and speed of the vehicle.

2) Growling Bear: The students put a capacitive sensor inside a small stuffed bear and connected the sensor to a CY3214 board. When a student stroked the bear, the bear would make a happy growling sound. If the bear was left alone for too long, it would make an angry growling sound.

3) Light Dimmer: The students used the CapSense slider on the CY3214 board to act as a light dimmer. As they slid their finger across the slider to the right, a row of LEDs would become brighter, and as they slid their finger across the slider to the left, the row of LEDs would become dimmer.

4) Relaxation Goggles: The students made a pair of relaxation goggles out of safety goggles, six LEDs, duct tape, and wire. They connected the LEDs on the goggles to the CY3214 board and allowed the user to control the rate at which the LEDS blinked by selecting different buttons on the board. These different rates corresponded to the dominant frequency of brain waves during states of deep sleep, rest, relaxation, and alertness.

5) Electronic Keyboard: The students made a single-octave keyboard out of copper tape mounted on Plexiglas. The copper tape "keys" acted as their own capacitive sensors. They connected the copper keys to the CY3214 board and programmed the board to play different notes when different keys were pressed. 


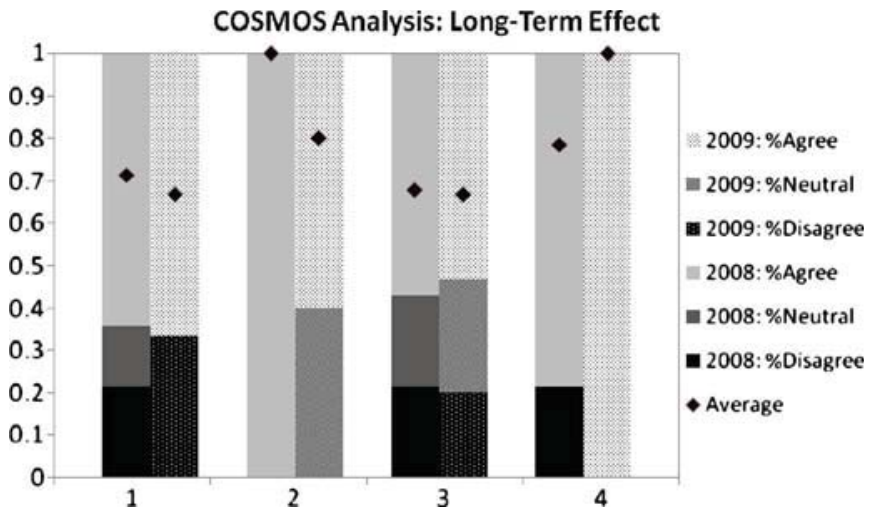

Fig. 2. COSMOS survey responses to the following questions: 1) My COSMOS experience helped me decide what field to pursue in college; 2) My experience in the COSMOS embedded systems cluster made me excited about embedded systems; 3 ) I would not have known anything (or known very little) about embedded systems had I not taken the COSMOS; 4) I described (plan to describe) my COSMOS experience on a college application, job application, or grant/scholarship application.

6) Stopwatch: The students created a stopwatch on the CY3214 board. One pushbutton acted as the start/stop button, and another as reset. The hour, minute, and second count appeared on the LCD.

The completion of all the labs and the success of the projects illustrate COSMOS did impart embedded systems knowledge to young students.

Fig. 2 reports the results of the survey questions related to enhancing young students' interests and opportunities in the field. The solid bars on the left show the responses from the 2008 class, and the patterned bars on the right show the responses from the 2009 class.

More than $50 \%$ of the students who responded to the survey from both years agreed that their COSMOS experience helped them decide what field to pursue in college, whether it be to continue in the field of computer science/engineering or to study a completely different field. Some of the students who reported a neutral response said that COSMOS made them more confused about what to study in college because it opened their eyes to more possibilities. Some of the students who disagreed with the statement said they already knew they wanted to pursue computer engineering or a related field before they started COSMOS.

Of the students from the 2008 class, $100 \%$ were in agreement with the statement that "My experience in COSMOS made me excited about embedded systems," and $100 \%$ of the students from the 2009 class were either in agreement with or neutral about the same statement. The stronger agreement from the 2008 class may have been due to the fact that they completed the survey 10 months after their course and looked back at the course with fond memories, whereas the students in the 2009 class completed the survey on their last day of the class when they were still having problems with their final projects.

More than $50 \%$ of the students from each year agreed that they would not have known anything (or known very little) about embedded systems had they not taken the COSMOS embedded systems course. This result illustrates that the COSMOS course provided the desired early exposure to embedded systems that most of the students would not have otherwise received.

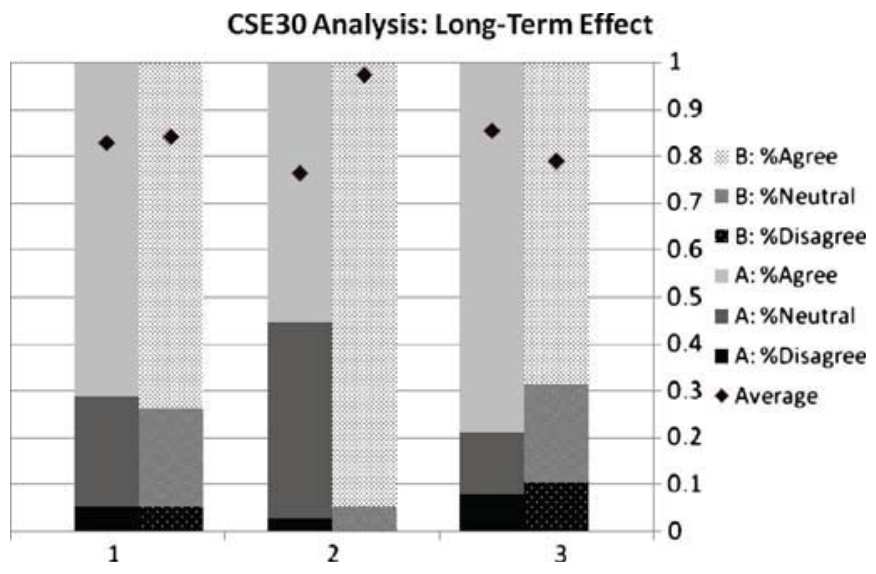

Fig. 3. CSE30 survey responses to the following questions: 1) CS30 increased my confidence in my decision to be a computer science or engineering major; 2) The programming assignments using Unix (The hands-on lab assignments using the microcontroller boards) got me excited about working with systems programming (embedded systems); 3) I can foresee describing an exercise or project I worked on in CS30 to a potential employer, scholarship, or grad school application.

Finally, the large majority of the students from the 2008 class had already described their COSMOS experience on a college application, job application, grant, or scholarship application at the time they had completed the survey. Six of those students started college in Fall 2010, attending: the University of California, Los Angeles (UCLA)-Computer Science; UCLA_Computer Engineering; UCSD_Computer Science; University of California, Berkeley-Electrical Engineering and Computer Science; Massachusetts Institute of Technology (MIT) - Computer Science. Also, at the time of the survey, all of the students who responded from the 2009 class planned to describe their COSMOS experience on a college application, job application, grant, or scholarship application. This result illustrates that COSMOS may play a role in securing a student's advancement in higher education.

\section{B. CSE30}

Just as in the COSMOS course, the students in the Winter and Spring 2009 CSE30-B classes successfully completed their lab assignments and final projects, thus demonstrating increased knowledge in the field of embedded systems. The final projects were of similar caliber to those of the COSMOS courses and thus are not described here. Fig. 3 reports the results for the survey questions related to assessing the effect of the CSE30 course on enhancing young students' interests and opportunities in the field. The solid bars on the left show the responses from CSE30-A (the course without the embedded systems component), and the patterned bars on the right show the responses from CSE30-B (the course with the embedded systems component). The text in parenthesis in the figure caption addresses how the question was posed differently to the CSE30-B class.

There was almost no difference in the percentage of agreement and disagreement to the statement "CSE30 increased my confidence in my decision to be a computer science major," suggesting that whether the course contained embedded systems material or not, the majority of students wished to continue to pursue their chosen major. 
The next statement was posed differently to the two courses to better reflect their specific course material: CSE30-A focused more on systems programming labs (using Unix, C, assembly, library routines, etc.), while CSE30-B focused more on embedded systems labs. The figure shows that students in the CSE30-B class were overall more excited about the course material than those in the CSE30-A class.

More than $60 \%$ of the students in CSE30-B responded that they could foresee describing an exercise or project they worked on in CSE30 to a potential employer, scholarship application, or grad school application, while almost $80 \%$ of the students in CSE30-A responded in a similar manner. It was expected that students in CSE30-B would have responded more positively to this statement as their hands-on assignments with the microcontroller boards would show knowledge of both hardware and software design. However, as this was the first time CSE30-B was offered, its inefficiencies may have hampered students in gaining the intended benefit of the course.

\section{DISCUSSION}

The assessment data illustrate that COSMOS was a success. The course made the large majority of students excited about embedded systems and exposed more than half the students to a field they would have otherwise known very little about. It helped the majority of the students decide what fields to pursue in college and provided a great experience for students to describe on their college, job, or scholarship applications. Although the outcome of the course was extremely positive, the course presented some valuable lessons about how to teach the course better in the future.

1) Programming Experience: In 2008, students who had no programming experience prior to the course struggled a lot more with the lab assignments and the projects. Although the lectures and workshops on programming were useful, it was impossible to expect a student to be able to write a full $\mathrm{C}$ program by the end of a two-week period without prior programming experience. Though the students still enjoyed the course, trying to learn programming and design small embedded systems at the same time was a bit overwhelming for them. The assessment data revealed that the majority of the students would have liked to have had more programming assignments, presumably to improve their mastery and understanding of programming concepts. However, it is impossible to spend more time on programming in just a four-week period; thus, some basic programming knowledge should be a prerequisite for the course so that students can focus more on embedded system concepts rather than on semantics of programming. The COSMOS staff focused harder on selecting students with some programming experience for the 2009 COSMOS. The results were better, though there was still a large discrepancy between those with programming experience and those without it. Unfortunately, this discrepancy is hard to correct in such a short time frame. However, the Scratch [17] programming environment did prove to be a useful tool in the 2009 class and thus will be used in future COSMOS programs to teach the basics of programming. Scratch allowed those with substantial programming experience to produce very interesting projects while giving those less experienced students an introduction to basic programming concepts.
2) Lab Assignments: In 2008, the first few labs contained step-by-step instructions on how to implement the desired embedded design. The instructors then asked the students to try reading through online tutorials on the third assignment to give them practice in learning how to find references and help for projects on their own. This proved to be an overoptimistic approach because the manuals and application notes the students were able to find often contained too much jargon, making the document inaccessible to neophytes. The instructors quickly determined that they had to keep all lab assignments at a level students could understand to keep them interested. This discrepancy was corrected for the most part in the 2009 COSMOS by creating six labs with step-by-step instructions, which resulted in a much smoother time for all students, though they still experienced their share of difficulties. This is to be expected and is likely largely unavoidable. Instructors also provided advanced exercises for each of the labs to make sure that the more advanced students were not "bored." This was very successful, and many students made enhancements above and beyond those that were suggested.

3) Lectures: As expected, limiting the lecture content and allowing for more hands-on work with the development boards proved more interesting and useful to the high school students. As related programs suggest, young students have a very short attention span, and trying to get material across to them in a conventional college lecture setting is difficult. Students were much more excited about going to lab and "playing" with the boards and were very quick to pick up concepts they experienced firsthand through experimentation rather than through lecture content. The assessment data suggests that the next COSMOS course should have even more lab assignments and fewer lectures, as illustrated by the students' strong agreement with the statement "I would have liked to spend more time working with the Cypress boards."

The assessment data also illustrate that the experiment with adding embedded systems concepts to the CSE30 class was somewhat successful, as the majority of students became excited about embedded systems as a result of working with the Cypress boards. However, the students' open-ended comments suggested that the course could be greatly improved with increased organization, more access to teaching assistants (TAs) or the instructor, and more hands-on lab experience with the development boards. As discovered in COSMOS, providing a hands-on lab experience for entry-level students requires a lot of support; at least two TAs per 20 students is necessary. In future course offerings, CSE30 will use the six step-by-step labs from the 2009 COSMOS as weekly projects. The students will be encouraged, but not required, to perform the follow-up enhancements and will be given bonus points for any additional work. Project work beyond this will not be included in CSE30 due to the lack of teaching resources. Project-based classes are a substantial undertaking and are difficult to manage for an introductory-level required course. A follow-up project-based class (CSE145) was offered for the first time in Spring 2010 to allow students to develop projects of their choosing in a small team environment. Enrollment in this class was limited to ensure an appropriate TA-to-student ratio.

The authors have continued to incorporate embedded systems into the high school and early undergraduate curriculum by assisting a local high school to set up a COSMOS-like course 
for the 2009-2010 academic school year, offering COSMOS Summer 2010, incorporating the COSMOS labs in all CSE30 classes starting Fall 2009, and offering CSE145 starting Spring 2010. This continued early exposure gave young students the interest and initiative to pursue an embedded systems education and take more advanced courses later in the curriculum to be better prepared for the demands of today's embedded world.

\section{ACKNOWLEDGMENT}

The authors thank Cypress Semiconductor and especially P. Kane, the Cypress Education Liaison, for their support of COSMOS and CS30. The authors also thank all the COSMOS students and staff, especially S. Miranda, Teacher Fellow, and J. Lunsford, Lab Manager, for helping to make the COSMOS program a great success.

\section{REFERENCES}

[1] J. Ganssle, "Embedded Y2K," Embedded Syst. Program., vol. 3, no. 17, pp. 97-99, 1999.

[2] D. J. Jackson and P. Caspi, "Embedded systems education: Future directions, initiatives, and cooperation," SIGBED Rev. vol. 2, no. 4, pp. 1-4, 2005.

[3] F. Vahid, F. , and T. Givargis, "Timing is everything —Embedded systems demand early teaching of structured time-oriented programming," in Proc. Workshop Embedded Syst. Educ., 2008, pp. 1-9.

[4] J. Turley, "The two percent solution," Embedded Syst. Program., vol. 16, no. 1, p. 29, 2003.

[5] G. C. Gannod, F. Golshani, B. Huey, Y. H. Lee, S. Panchanathan, and D. Pheanis, "A consortium-based model for the development of a concentration track in embedded systems," in Proc. Amer. Soc. Eng. Educ. Апnи. Conf. Expos., 2002, p. 1532.

[6] W. Wolf, "Rethinking embedded microprocessor education," in Proc. Amer. Soc. Eng. Educ. Annu. Conf. Expos., Albuquerque, NM, 2001.

[7] “UCSD COSMOS program," UCSD, La Jolla, CA, Sep. 10, 2010 [Online]. Available: http://www.jacobsschool.ucsd.edu/cosmos/

[8] T. Mitra, "Challenges in designing embedded systems courses," in Proc. Workshop Embedded Syst. Educ., 2006, pp. 1-4.

[9] P. O. Bobbie, J. Uboh, and B. Davis, "A project in embedded system design and development: A partnership with area high schools," in Proc. Frontiers Educ. Conf., Savannah, GA, 2004, pp. F4D-14-F4D-17.

[10] "Embedded software actual condition survey," Japanese Ministry of Economy Trade and Industry,, Japan, 2006 [Online]. Available: https:// sec.ipa.go.jp/download/200606es.php

[11] Y. Matsubara, M. Sugaya, I. Taniguchi, Y. Murakami, H. Kanai, and H. Takada, "SSEST: Summer school on embedded system technologies," in Proc. Int. Conf. Parallel Distrib. Syst., 2007, vol. 2, pp. 1-8.

[12] “LEGO Mindstorms," LEGO, Billund, Denmark, Sep. 10, 2010 [Online]. Available: http://mindstorms.lego.com

[13] "Institute for Personal Robots in Education (IPRE)," USA, Sep. 10, 2010 [Online]. Available: http://www.roboteducation.org/

[14] "Cypress Semiconductor PSoC CapSensePLUS With USB Evaluation Kit (CY3214 Development Kit) \& CY3209 Express Evaluation Kit," Cypress, San Jose, CA, Sep. 10, 2010 [Online]. Available: http://www. cypress.com/

[15] M. Guzdial, "A media computation course for non-majors," in Proc. 8th Annu. Conf. Innov. Technol. Comput. Sci. Educ., Thessaloniki, Greece, 2003, pp. 104-108.

[16] R. Likert, "A technique for the measurement of attitudes," Arch. Psych., vol. 140, pp. 1-55, 1932

[17] "Scratch," MIT, Cambridge, MA, Sep. 10, 2010 [Online]. Available: http://scratch.mit.edu/

Bridget Benson (M'10) received the B.S. degree in computer engineering from California Polytechnic State University, San Luis Obispo, in 2005; the M.S. degree in electrical and computer engineering from the University of California, Santa Barbara, in 2007; and the Ph.D. degree in computer science and engineering from the University of California, San Diego, La Jolla, in 2010.
She is currently a Post-Doctoral Researcher with the Center for Coastal Environmental Sensing Networks, University of Massachusetts, Boston. Her research interests span computer engineering and aquatic sciences, focusing on selecting and developing technology to advance marine science research.

Arash Arfaee received the B.S. degree in computer hardware engineering from Azad University, South Tehran, Iran, in 2001, and the M.S. degree in computer engineering from Iran University of Science and Technology, Tehran, Iran, in 2002. He is currently pursuing the Ph.D. degree with the Department of Computer Science and Engineering, University of California, San Diego, La Jolla.

His research interests lie in computer hardware security.

Choon Kim received the B.S. degree in industrial education from Seoul National University, Seoul, Korea, in 1976, and the M.S. and Ph.D. degrees in electrical engineering from the University of Minnesota, Twin Cities, in 1982 and 1986, respectively.

$\mathrm{He}$ is as a Senior Development Engineer with the Computer Science and Engineering Department, University of California, San Diego (UCSD), La Jolla, developing an Advanced Embedded System Laboratory for research and education. He also has been teaching a LAB-based practical embedded system design course in the UCSD extension program for local engineers. He has more than 20 years experience in digital ASIC design in wireless communications. He has worked at companies including Qualcomm, General Instrument, PCSI, and Intergraph Corporation.

Ryan Kastner (S'00-M'02) received two B.S. degrees in electrical engineering and computer engineering and the M.S. degree in engineering from Northwestern University, Evanston, IL, in 1999 and 2000, respectively, and the Ph.D. degree in computer science from the University of California, Los Angeles, in 2002.

He is an Associate Professor with the Department of Computer Science and Engineering, University of California, San Diego, La Jolla. He spent the first five years after receiving the Ph.D. degree as a Professor with the Department of Electrical and Computer Engineering, University of California, Santa Barbara. He has published more than 100 technical articles and has authored three books: Synthesis Techniques and Optimizations for Reconfigurable Systems (Springer, 2003), Arithmetic Optimizations for Polynomial Expressions and Linear Systems (Cambridge Univ. Press, 2010), and Handbook on FPGA Design Security (Springer, 2010). His current research interests reside in the realm of embedded system design - in particular, the use of reconfigurable computing devices for digital signal processing as well as hardware security.

Prof. Kastner serves on the Editorial Board of IEEE EMBEDDED SYSTEMS LETTERS. He has served as a member of numerous conference technical committees spanning topics like reconfigurable computing (ISFPGA, FPL, FPT, ERSA, RAW, ARC), electronic design automation (DAC, ICCAD, DATE, ICCD, GLSVLSI), wireless communication (GLOBECOM, SUTC), hardware security (HOST), and underwater networking (WUWNet).

Rajesh K. Gupta (F'08) received the B.Tech. degree in electrical engineering from the Indian Institute of Technology, Kanpur, Kalyanpur, India, in 1984; the M.S. degree in electrical engineering and computer science from the University of California, Berkeley, in 1986; and the Ph.D. degree in electrical engineering from Stanford University, Stanford, CA, in 1994.

$\mathrm{He}$ is a Professor of computer science and engineering at the University of California, San Diego (UCSD), La Jolla, and holds the Qualcomm endowed chair. He directs the smart buildings/smart grids task force at UCSD in his role as Associate Director for the California Institute for Telecommunications and Information Technology (CalIT2). His recent contributions include SystemC modeling and SPARK parallelizing high-level synthesis, both of which are publicly available and have been incorporated into industrial practice. Earlier, he led or co-led DARPA-sponsored efforts under the Data Intensive Systems (DIS) and Power Aware Computing and Communications (PACC) programs that demonstrated architectural adaptation and compiler optimizations in building high-performance and energy-efficient system architectures. His ongoing efforts include energy-efficient data-centers and large-scale computing using memory-coherent algorithmic accelerators and nonvolatile storage systems. His research interests are in energy-efficient systems that have taken a turn toward large-scale energy use in recent years.

Prof. Gupta currently serves as Editor-in-Chief of IEEE EMBEDDED SYSTEMS LETTERS. He and his students have received a Best Paper Award at IEEE/ACM DCOSS 2008 and a Best Demonstration Award at IEEE/ACM IPSN/SPOTS 2005 . 